\title{
Toward an AIDS vaccine: lessons from natural simian immunodeficiency virus infections of African nonhuman primate hosts
}

\author{
Donald L Sodora $^{1,15}$, Jonathan S Allan ${ }^{2,15}$, Cristian Apetrei ${ }^{3,4,15}$, Jason M Brenchley ${ }^{5,15}$, Daniel C Douek ${ }^{6,15}$, \\ James G Else ${ }^{7,15}$, Jacob D Estes ${ }^{8,15}$, Beatrice H Hahn ${ }^{9,15}$, Vanessa M Hirsch ${ }^{5,15}$, Amitinder Kaur ${ }^{10,15}$, \\ Frank Kirchhoff ${ }^{11,15}$, Michaela Muller-Trutwin ${ }^{12,15}$, Ivona Pandrea ${ }^{3,4,15}$, Jörn E Schmitz ${ }^{13,15}$ \& Guido Silvestri ${ }^{7,14,15}$
}

The design of an effective AIDS vaccine has eluded the efforts of the scientific community to the point that alternative approaches to classic vaccine formulations have to be considered. We propose here that HIV vaccine research could greatly benefit from the study of natural simian immunodeficiency virus (SIV) infections of African nonhuman primates. Natural SIV hosts (for example, sooty mangabeys, African green monkeys and mandrills) share many features of HIV infection of humans; however, they usually do not develop immunodeficiency. These natural, nonprogressive SIV infections represent an evolutionary adaptation that allows a peaceful coexistence of primate lentiviruses and the host immune system. This adaptation does not result in reduced viral replication but, rather, involves phenotypic changes to $\mathrm{CD}^{+} \mathrm{T}$ cell subsets, limited immune activation and preserved mucosal immunity, all of which contribute to the avoidance of disease progression and, possibly, to the reduction of vertical SIV transmission. Here we summarize the current understanding of SIV infection of African nonhuman primates and discuss how unraveling these evolutionary adaptations

${ }^{1}$ Seattle Biomedical Research Institute, Seattle, Washington, USA. ${ }^{2}$ Southwest National Primate Research Center, San Antonio, Texas, USA. ${ }^{3}$ Tulane

National Primate Research Center, Tulane University, Covington, Louisiana, USA. ${ }^{4}$ Center for Vaccine Research, University of Pittsburgh, Pittsburgh, Pennsylvania, USA. ${ }^{5}$ Laboratory of Molecular Microbiology, National Institute of Allergy and Infectious Diseases, US National Institutes of Health, Bethesda, Maryland, USA. ${ }^{6}$ Vaccine Research Center, ${ }^{7}$ Yerkes National Primate Research Center, Atlanta, Georgia, USA. ${ }^{8}$ US National Cancer Institute, Frederick, Maryland, USA. ${ }^{9}$ Departments of Microbiology and Medicine, University of Alabama, Birmingham, Alabama, USA. ${ }^{10}$ New England Primate Research Center, Harvard Medical School, Southborough, Massachusetts, USA.

${ }^{11}$ Institute of Molecular Virology, University of UIm, UIm, Germany. ${ }^{12}$ Institut

Pasteur, Unite de Regulation des Infections Retrovirales, Paris, France.

${ }^{13}$ Division of Viral Pathogenesis, Beth Israel Deaconess Medical Center, Harvard Medical School, Boston, Massachusetts, USA. ${ }^{14}$ Department of Pathology and Laboratory Medicine, University of Pennsylvania, Philadelphia, Pennsylvania, USA. ${ }^{15}$ All authors contributed equally to this manuscript.

Correspondence should be addressed to G.S. (gsilvest@mail.med.upenn.edu).

Published online 06 August 2009; doi:10.1038/nm.2013 may provide clues for new vaccine designs that might induce effective immune responses without the harmful consequences of excessive immune activation.

Over 25 years after the discovery of HIV as the etiologic agent of AIDS, no effective vaccine for the disease is available. Indeed, the two large-scale clinical trials (AIDSVax and STEP) aimed at testing proof-of-principle vaccines that were based on the induction of HIV-specific antibodies and cytotoxic T cell responses, respectively, failed to show any of the desired clinical efficacy ${ }^{1}$. The reasons why an effective AIDS vaccine is not available are complex and, for the most part, are related to specific features of primate lentiviruses and their interaction with the host immune system. First, HIV establishes latency, that is, a persistent infection of cellular reservoirs that do not produce virions and thus are immunologically silent; thus, HIV is virtually never cleared by host immune responses ${ }^{2}$. Second, the HIV reverse transcriptase is an 'error-prone' enzyme that generates markedly diverse and constantly evolving virus populations within infected individuals ${ }^{3}$. This genetic diversity is fueled by high replication rates that lead to the rapid generation of viral variants that can escape the host immune responses. Third, HIV preferentially infects activated $\mathrm{CD} 4^{+} \mathrm{T}$ helper cells expressing the CCR 5 co-receptor ${ }^{4}$. This latter point is of great importance because it raises the possibility that HIV-specific adaptive immune responses induced by a candidate AIDS vaccine can generate more targets for the virus and thus paradoxically facilitate HIV acquisition and disease progression. For these reasons, it is possible that conventional immunization strategies (those based solely on the elicitation of high levels of antiviral neutralizing antibodies and cytotoxic $\mathrm{T}$ lymphocyte (CTL) responses) may not be sufficient to provide protection against HIV infection and AIDS. Here we discuss how insights gained from studies of nonhuman primates naturally infected with SIV may uncover unconventional strategies to enhance the efficacy of conventional AIDS vaccine approaches.

\section{Natural SIV infections}

Over 40 nonhuman primate species in Sub-Saharan Africa are infected in the wild with species-specific lentiviruses collectively termed SIV 5 These infections represent virus-host relationships that are evolutionarily older than HIV infection of humans. Phylogenetic data indicate that cross-species transmissions of SIV from two of these natural hosts, chimpanzees (SIVcpz) and sooty mangabeys (SIVsmm), to humans have generated HIV-1 and HIV-2, respectively ${ }^{5,6}$. Thus far, the majority of 
Table 1 Similarities and differences between well-adapted natural SIV hosts and poorly adapted recent or experimental HIV and SIV hosts.

\author{
Well-adapted natural SIV hosts \\ (sooty mangabeys and African green monkeys) \\ Acute infection \\ Virus replication peaks within a few weeks from infection. \\ Post-peak decline of viral load indicates partial control of virus replication. \\ Elevated innate and adaptive immune response to the virus. \\ Marked type I interferon response. \\ Variable levels of bystander T cell activation and apoptosis. \\ Establishment of an anti-inflammatory milieu. \\ Depletion of mucosal $C D 4^{+} T$ cells.
}

\section{Chronic infection}

High set point of virus replication that persists despite the host immune response.

Virus replication mostly occurring in short-lived, activated $C D 4^{+} \mathrm{T}$ cells. Healthy $\mathrm{CD}^{+}{ }^{+} \mathrm{T}$ cell counts in most animals. No progressive depletion of mucosal $\mathrm{CD}^{+}{ }^{+} \mathrm{T}$ cells.

Resolution of type I interferon responses.

Limited immune activation and $\mathrm{T}$ cell apoptosis.

Preservation of mucosal $\mathrm{T}_{H} 17$ cells.

Absence of microbial translocation.

\section{Clinical outcome}

Progression to AIDS is very rare.

Vertical transmission is rare.

studies in natural primate hosts have been conducted in just three species, sooty mangabeys, African green monkeys (AGMs) and mandrills ${ }^{7-9}$, whereas SIV infection of other natural host species has been investigated only at a cursory level or has not yet been assessed. A major focus has been to investigate immunologic, virologic and clinical parameters in these natural SIV infections and to compare them to pathogenic HIV-1 infection of humans and SIVmac infection of macaques. These studies have uncovered a number of similarities between pathogenic and nonprogressive primate lentivirus infections, as well as a series of features unique to the natural SIV infections that have provided new clues as to why they remain nonpathogenic (Table 1).

The most salient feature of natural SIV infections is the general lack of progression to AIDS. Although a few cases of simian AIDS have been described in naturally or experimentally infected AGMs, sooty mangabeys and mandrills, these instances are exceedingly rare ${ }^{10}$. Nonetheless, these examples of disease progression demonstrate that the corresponding viruses have retained some of their pathogenic potential, even though the host is able to counteract it for a time period that generally exceeds the lifespan of the animal ${ }^{10}$. We believe that this balancing act is the result of a long-standing evolutionary coadaptation between primate lentiviruses and their hosts' immune systems. The recent observation that SIVcpzinfected chimpanzees have a substantially higher mortality rate (10- to 16-fold increased death hazard) than their uninfected counterparts ${ }^{11}$ supports this hypothesis, as these apes acquired their infection more recently by cross-species transmission of SIVs infecting the monkeys on which they prey ${ }^{12}$. Of note, a moderately pathogenic outcome is also observed after HIV-2 infection, which originated from cross-species transmission of SIVsmm from sooty mangabeys to humans ${ }^{6}$. Further studies in SIVcpz-infected chimpanzees and HIV-2-infected individuals will be essential to identify similarities and differences with both the typically nonprogressive infections of natural hosts and the more aggressive HIV-1 and SIVmac infections of humans and macaques.

Despite their resistance to disease progression, SIV infections of sooty mangabeys, AGMs and mandrills show numerous similarities with pathogenic infections, as illustrated in Table 1, thus underscoring their relevance to AIDS research. Common features of natural and pathogenic
Nonadapted recent or experimental hosts

(humans and Asian macaques)

Acute infection

Virus replication peaks within a few weeks from infection.

Post-peak decline of viral load indicates partial control of virus replication.

Elevated innate and adaptive immune response to the virus.

Marked type I interferon response.

High levels of bystander $\mathrm{T}$ cell activation and apoptosis.

Depletion of mucosal CD4+ $\mathrm{T}$ cells.

Chronic infection

High set point of virus replication that persists despite the host immune response. Virus replication mostly occurring in short-lived, activated CD4 ${ }^{+} \mathrm{T}$ cells.

Progressive depletion of circulating and mucosal $\mathrm{CD} 4^{+} \mathrm{T}$ cells.

Persistent type I interferon responses.

Generalized immune activation and significant $\mathrm{T}$ cell apoptosis.

Preferential loss of mucosal $\mathrm{T}_{\mathrm{H}} 17$ cells.

Microbial translocation is prominent.

Clinical outcome

Progression to AIDS occurs in most cases.

Vertical transmission is more common.

HIV and SIV infections include the following characteristics: the acute infection phase is characterized by a peak of virus replication, increased levels of many innate and adaptive immune responses, and depletion of mucosal CD4 ${ }^{+} \mathrm{T}_{\text {cells }}{ }^{13-19}$; the chronic phase of the infection is associated with persistently high levels of virus replication that are similar to those observed during pathogenic infections ${ }^{20-24}$; the bulk of virus replication occurs in short-lived activated CD4 ${ }^{+} \mathrm{T}_{\text {cells }}{ }^{25,26}$; and SIV-specific cellular and humoral immune responses are unable to fully suppress virus replication $^{18,27-30}$ (T. Gaufin, M. Pattison, R. Gautam, C. Stoulig, J. Dufour et al., unpublished data). These similarities notwithstanding, natural SIV infections also have key distinctive features that clearly set them apart from pathogenic HIV and SIV infections (Tables 1 and 2). These include a benign clinical course, a relative rarity of vertical transmissions, the maintenance of peripheral $\mathrm{CD} 4^{+} \mathrm{T}$ cell counts at normal levels in the majority of animals, the absence of chronic immune activation, the preservation of mucosal immunity with normal levels of mucosal T helper type 17 $\left(\mathrm{T}_{\mathrm{H}} 17\right)$ cells and absence of microbial translocation ${ }^{14,16,17,22-24,31-34}$. Thus, comparative studies of natural, nonprogressive hosts provide a unique opportunity to elucidate the mechanisms that underlie disease progression in hosts that show pathogenic infections (that is, humans and macaques).

\section{Antiviral immune responses in natural SIV hosts}

The entire field of AIDS vaccinology is currently based on the premise that a strong adaptive immune response to HIV and SIV will decrease virus replication and disease progression. Theoretically, the fact that natural hosts of SIVs do not develop AIDS could be the result of adaptive immune responses (both humoral and cellular) that are significantly more effective than those mounted against pathogenic HIV and SIV infections of humans and macaques. However, one would expect that, if this hypothesis is correct, the levels of virus replication would be much lower than those observed in pathogenic HIV and SIV infections. On the contrary, the natural hosts investigated so far show levels of virus replication comparable to or higher than those observed during pathogenic HIV and SIV infections ${ }^{7-9}$. Although in all cases of SIV infection of natural hosts, a partial containment of viremia has been observed during the 
transition between acute and chronic infection, the relatively high set point level of viral replication does not support the notion of potentially more effective adaptive immune responses in natural hosts.

To assess more directly the potential role of adaptive immune responses in viral containment, a number of recent studies have used monoclonal antibody treatments to deplete lymphocyte subsets in vivo. In nonnatural hosts (macaques), the central role of $\mathrm{CD} 8^{+}$lymphocytes was documented in numerous studies showing that depletion of these cells results in increased virus replication and accelerated disease progres$\operatorname{sion}^{35}$. The impact of $\mathrm{CD}^{+}$lymphocyte depletion on plasma viremia in natural hosts has generally been either less impressive ${ }^{36}$ or similar to that observed in SIV-infected macaques (C.A., I.P., T. Gaufin, M. Pattison, R. Gautam et al., unpublished data; J.E.S., R.C. Zahn, M.D. Rett, M. Li, H. Tang et al., unpublished data; A.K., Z. Wang, N. Kassis, R.M. Ribeiro, S. Pryputniewicz et al., unpublished data). Similarly, SIV-specific CD8 ${ }^{+}$ $\mathrm{T}$ cell responses in natural SIV infections were found to be comparable or lower in breadth and magnitude than those reported in pathogenic HIV and SIV infections ${ }^{18,27-29,30}$. SIV-specific antibodies have also been documented in natural host infections, with both binding or neutralizing antibodies present at levels that are either similar to or lower than those observed in SIVmac-infected macaques and HIV-1-infected humans ${ }^{37,38}$ (T. Gaufin, M. Pattison, R. Gautam, C. Stoulig, J. Dufour et al., unpublished data). Notably, B cell depletion experiments were not associated with marked increases in viral replication or clinical signs of disease $\mathrm{s}^{30}$ (T. Gaufin, M. Pattison, R. Gautam, C. Stoulig, J. Dufour et al., unpublished data; R.C. Zahn, M.D. Rett, M. Li, H. Tang, B. Korioth-Schmitz et al., unpublished data), suggesting a limited role of humoral immune responses in viral containment of SIV infections. The only consistent feature of the virus-specific humoral immune response in natural SIV hosts is a paucity of Gag-specific antibodies; however, the biological role of this finding remains to be determined. Collectively, these data indicate that natural SIV hosts generate virus-specific cellular and humoral responses that are lower than or comparable to those elicited by pathogenic HIV and SIV infections and similarly unable to suppress virus replication fully. However, more detailed investigations are required to better understand the contribution and possible limitations of adaptive immune responses in natural hosts. When translated in terms of AIDS vaccine research, the insights that have been obtained so far from the study of adaptive immune responses in natural SIV infections would argue that a new paradigm for the control of HIV disease progression should be explored, one that does not solely depend on conventional immunization strategies with viral immunogens.

\section{SIV transmission in natural hosts}

Relatively little is known about the routes of SIV transmission in natural hosts, especially when compared to the wealth of information available for HIV transmission in humans. The high prevalence rates of SIV in adult sooty mangabeys, AGMs and mandrills both in captivity and in the wild ${ }^{5}$ suggest that horizontal transmission (either through sexual contacts or aggressive behavior) is common. However, it is currently impossible to compare the actual frequency of these transmissions with those reported for HIV-1 infection in humans, as all infections of sooty mangabeys, AGMs and mandrills in captivity were administered intravenously. Interestingly, vertical transmission in natural SIV hosts seems to be rare ${ }^{38-40}$, with an observed frequency of mother-to-offspring transmissions of SIV in naturally infected sooty mangabeys below $5 \% 38$ (T. Meeker, J.G.E. and G.S., unpublished data). The reasons for this phenomenon remain to be elucidated; however, recent data suggest that, at

\section{Table 2 How studies of natural SIV hosts may inform AIDS vaccine development: proposed research priorities}

\begin{tabular}{|c|c|}
\hline AIDS prevention strategies & Lessons from natural SIV hosts \\
\hline $\begin{array}{l}\text { Identify neutralizing antibodies in } \\
\text { naturally infected primates that target } \\
\text { epitopes (for example, CD4 binding site } \\
\text { or member-proximal external region) } \\
\text { that are highly conserved among primate } \\
\text { lentiviruses. }\end{array}$ & $\begin{array}{l}\text { Lifelong nonpathogenic SIV infection } \\
\text { may elicit neutralizing antibodies-in } \\
\text { the absence of B cell hyperactivation }{ }^{34} \\
\text { - that are qualitatively and quantita- } \\
\text { tively different from those produced in } \\
\text { HIV-1 infection. }\end{array}$ \\
\hline $\begin{array}{l}\text { Induce virus-specific cellular immune } \\
\text { responses that can delay disease } \\
\text { progression. }\end{array}$ & $\begin{array}{l}\text { SIV-specific T cell responses are similar } \\
\text { in magnitude and breadth but are not } \\
\text { associated with bystander T cell activa- } \\
\text { tion } 18,27-29,30 \text {. }\end{array}$ \\
\hline $\begin{array}{l}\text { Maximize the antiviral activity of } \\
\text { innate immune responses using } \\
\text { vaccine adjuvants. }\end{array}$ & $\begin{array}{l}\text { Innate immune responses and type I } \\
\text { interferon responses are robust in acute } \\
\text { but not chronic infection }{ }^{13-19,37 .}\end{array}$ \\
\hline
\end{tabular}
vaccine adjuvants.

Decrease the availability of CD4 ${ }^{+} \mathrm{CCR} 5^{+}$ target cells at the site of virus transmission

Reduce the level of chronic immune activation in the event of breakthrough infection.

\section{Elevated immune activation in acute} infection is rapidly downregulated $\operatorname{sion}^{46}$.

Prevent mother-to-child transmission (MTCT).
Natural SIV hosts express low amounts of CCR5 on $\mathrm{CD}^{+}{ }^{+} \mathrm{T}$ cells ${ }^{41,42}$. AGMs downmodulate CD4 expression upon $\mathrm{T}$ cell activation 45 . during chronic infection 15,18,42.

Natural SIV hosts preserve mucosal immunity and gut $T_{H} 17$ cells and avoid microbial translocation despite depletion of mucosal CD4 ${ }^{+} \mathrm{T}$ cells ${ }^{16}$, 17,32,37.

HIV-1 Nef has lost the ability to downmodulate CD3-T cell receptor expres-

Key future research priorities

Assess neutralizing antibody responses in natural SIV infections, determine level of cross-reactivity and define the basis for the lack of B cell hyperactivation.

Identify the link between SIV-specific

$T$ cell responses and T cell hyperactiva-

tion in pathogenic and nonpathogenic

HIV and SIV infections.

Define the mechanisms by which innate immune responses to SIV are rapidly downmodulated in natural SIV hosts.

Elucidate the mechanisms underlying the differing expression of CD4, CCR5 or both in natural SIV hosts.

Identify the mechanisms responsible for the lack of chronic immune activatio in natural SIV hosts: immunomodulatory pathways (T regulatory cells, the negative regulator PD-1 and transforming growth factor- $\beta$, among others); specific virus proteins (Nef, Vpu and Env); and preserved mucosal immunity and lack of microbial translocation. Assess speciesspecific differences in innate and adaptive responses to SIV.

MTCT of SIV is rare in natural SIV hosts $^{38-40}$
Define the mechanisms underlying the partial resistance to MTCT in natural SIV hosts (maternal or fetal antiviral factors; role of low CCR5 expression and CD4 downmodulation).
Potential immunization strategies

Design immunoadhesins using components of SIV-specific neutralizing antibodies from naturally infected species and deliver them to mucosal surfaces using vectors (for example, adeno-associated virus vector).

Develop immunization approaches that induce strong HIV-specific T cel responses without inducing long-term, chronic-stage bystander T cell activation.

Develop adjuvants that, when coupled with an immunogen, result in strong but transient innate immune responses to HIV.

Couple CCR5 antagonists with immunogens to induce HIV-specific responses that are independent of activated CD4 ${ }^{+}$CCR $5{ }^{+} T$ cells at mucosal tissues.

Develop immunogens that have built-in factors to prevent the development of chronic immune activation in the event of HIV infection.

Develop immunization approaches that stimulate the negative control of inflammation in the event of HIV infection.

Develop immunogenic approaches that mimic the mechanisms underlying the relative resistance to $\mathrm{MTCT}$ in natural SIV hosts. 
least in mandrills and AGMs, low CCR5 expression on CD4 ${ }^{+} \mathrm{T}$ cells in the offspring correlates with a resistance to SIV transmission via breastfeeding ${ }^{40}$. Regardless of the mechanism(s), the observation that SIV is not easily transmitted from mother to infant in natural SIV hosts is intriguing because it implies an evolutionary pressure against this type of infection. Whatever the reasons, we believe that understanding the mechanisms underlying SIV transmission in natural hosts, including the role of the observed low levels of activated $\mathrm{CD} 4^{+} \mathrm{CCR} 5^{+} \mathrm{T}$ cells in fetuses and newborns, may provide crucial clues for a better understanding of HIV transmission in humans. In addition, reduced CCR5 expression on CD4 ${ }^{+}$ T cells ${ }^{41}$ and low immune activation upon SIV infection may represent evolutionary adaptations that make the mucosal milieu of natural SIV hosts more resistant to virus transmission in a way that is independent of, or perhaps complementary to, classical adaptive antiviral immunity.

\section{Features of natural SIV hosts that might help vaccine design}

The unique features of natural SIV infections have been the subject of a number of recent reviews on the topic ${ }^{7-9}$. Here we focus on those features that we believe can be exploited for new vaccine strategies. There are two types of conventional HIV vaccine approaches currently being explored: HIV-specific CTL-based vaccines designed to delay disease progression by killing infected target cells and HIV-specific antibodybased vaccines designed to inhibit HIV transmission. We believe that studies of natural SIV infections point to additional avenues that should be exploited in an attempt to increase protection against both infection and disease progression.

Natural SIV infections are characterized by levels of chronic immune activation that are consistently and substantially lower than those observed in pathogenic HIV and SIV infections ${ }^{14-18,23,24,31,36,37,42}$. Notably, both innate and adaptive immune activation are observed during the acute phase of SIV infection of natural hosts ${ }^{16-18,37,42}$, including strong upregulation of type I interferon-stimulated genes in both peripheral blood and lymph nodes ${ }^{19}$ (J.G.E., G.S., S. Bosinger, S.N. Gordon, N.R. Klatt et al., unpublished data; Li, Q., Duan, L., Smith, A.J., Zeng, M., Masopust, D. et al., unpublished data; M.M.-T., B. Jacquelin, V. Mayau, B. Targat, A.S. Liovat et al., unpublished data) as well as type I interferon production by plasmacytoid dendritic cells in lymph nodes (J.D.E., L. Harris, S.N. Gordon, G.S. and J.M.B., unpublished data). However, marked differences in the levels of immune activation between natural and non-natural HIV and SIV hosts are observed after the transition from the acute to the chronic phase of infection. This finding suggests the presence of active immune downmodulatory mechanisms rather than an intrinsically attenuated innate immune response to SIV infection $15,17,40,43$. This conclusion is also supported by recent studies in which immune activation and $\mathrm{CD} 4^{+} \mathrm{T}$ cell depletion were successfully induced in AGMs after administration of lipopolysaccharide ${ }^{44}$. Several properties of the AGM and sooty mangabey hosts that may contribute to the low immune activation during chronic infection have recently been identified and warrant further investigation, particularly with regard to the association between increased immune activation and effective SIV-specific adaptive immune responses. These potential immunomodulatory mechanisms include the rapid establishment of an anti-inflammatory milieu, the early upregulation of the negative regulator PD-1 in lymph node-derived T cells, a partial refractoriness of plasmacytoid dendritic cells to produce type I interferon in response to SIV RNA, absence of microbial translocation from the intestinal lumen to the systemic circulation, low levels of CCR 5 expression on $\mathrm{CD} 4^{+} \mathrm{T}$ cells and downregulation of CD4 expression by T cells ${ }^{15,17,33,40,42,43,45}$. In addition, lower immune activation in natural hosts may also be associated with virus-specific properties, particularly the lack of a $v p u$ gene and the ability of the accessory Nef protein to suppress the activation of virally infected $\mathrm{T}$ cells $\mathrm{s}^{46,47}$. The absence of chronic hyperimmune activation may be crucial to preserve $\mathrm{T}$ cell homeostasis (particularly at the level of naive and central memory cells), maintain normal lymphoid tissue architecture, preserve non-T cell immune functions, including those of antigen-presenting cells, and allow the natural host to better cope with instances of mucosal and generalized $\mathrm{CD}^{+}{ }^{+} \mathrm{T}$ cell depletion $^{16,17,34,37,48}$.

The low numbers of $\mathrm{CD} 4^{+} \mathrm{CCR} 5^{+}$target cells observed in SIV natural hosts represent a striking contrast to what has been reported in Asian macaques and humans ${ }^{41}$. However, the biological relevance of this observation is still unclear-especially because these low amounts of CCR5 on $\mathrm{CD}^{+} \mathrm{T}$ cells do not result in lower virus replication in natural hosts or preclude $\mathrm{CD}^{+} \mathrm{T}$ cells from being the main source of virus production in natural SIV infections ${ }^{8,25,26,49}$. It is conceivable that, in natural SIV hosts, a restriction of CCR5 expression to more activated effector and/or CD4 ${ }^{+}$ T cells may confine SIV replication to a subset of more 'expendable' cells, thus better preserving the homeostasis of central memory $\mathrm{CD} 4^{+} \mathrm{T}$ cells despite similarly high levels of viremia. Low CCR 5 expression may also reduce the homing of activated $\mathrm{CD} 4^{+} \mathrm{T}$ cells to inflamed tissues, thus eliciting an anti-inflammatory effect ${ }^{41}$. Finally, a paucity of $\mathrm{CD} 4^{+} \mathrm{CCR} 5^{+}$ $\mathrm{T}$ cells in mucosal tissues may also influence SIV transmission, as it may affect the overall receptivity of the mucosal microenvironment to the establishment and early dissemination of the transmitted virus. Thus, a successful HIV vaccine may have to circumvent $\mathrm{CD} 4^{+} \mathrm{CCR} 5^{+} \mathrm{T}$ cell activation, particularly at mucosal sites that are important for HIV transmission. Alternatively, it might be advantageous if an HIV vaccine induces $\mathrm{T}$ cell populations that can provide the proper $\mathrm{T}$ cell helper function but do not express the CD4 receptor and therefore are refractory to HIV infection $\left(\mathrm{CD}^{+} \mathrm{CD} 4^{\text {neg }}\right)^{45}$ (C.A., T. Gaufin, E. Blake, R. Gautam and I.P. Pattison, unpublished data).

\section{Natural SIV hosts and AIDS vaccine design: a new paradigm}

Given these specific features of natural SIV hosts, it seems clear that a more complete understanding of the mechanisms that have enabled natural SIV infections to avoid disease progression may provide major clues for the design of successful AIDS vaccines. We know that SIV-infected natural hosts elicit innate and adaptive immune responses to the virus; however, these responses are unable to stop virus replication. We also know that natural host species combine their antiviral innate and adaptive immune responses with low overall immune activation during the chronic phase, which may be a key in achieving an environment that is less conducive to immunodeficiency. As such, these observations suggest that designing vaccines that focus solely on increasing adaptive immune responses to HIV, similar to more conventional vaccine approaches, may not be sufficient to inhibit infection, progression to AIDS or both. Recently, a study by Phil Johnson and his colleagues explored a unique vaccine approach involving vector-mediated gene transfer to express SIVspecific antibodies or immunoadhesins ${ }^{50}$. This study provides insights above and beyond this particular approach, as it illustrates how one might design additional novel, 'out-of-the-box' approaches to elicit protection from HIV transmission, disease progression or both. In this regard, studies of SIV-infected natural hosts have provided a number of insights as to how evolutionary pressure resulted in the ability to resist progression to AIDS and suggest that this information will help the design of future new vaccine approaches.

In Table 2, we have defined six different AIDS prevention strategies, and for each of them, we have outlined lessons from the natural SIV hosts, key future research priorities and potential immunization strategies. For example, one potential new vaccine approach that might originate from studies of natural hosts may consist of decreasing the availability of target cells for the virus at the level of mucosal tissues (Table 2). This goal could 
be achieved either by limiting the number of activated $\mathrm{CD} 4^{+} \mathrm{CCR} 5^{+}$cells or by increasing the levels of $\mathrm{CD} 3^{+} \mathrm{CD} 4^{\text {neg }}$ cells that retain functions similar to $\mathrm{CD}^{+} \mathrm{T}$ helper cells. In addition, natural hosts seem to have a lower incidence of mother-to-offspring transmission (Table 2), suggesting that natural SIV hosts may help in developing vaccine strategies aimed at limiting virus transmission in addition to those aimed at inhibiting progression to clinical AIDS. We also believe that the type of adjuvant used in any future HIV vaccine, whether antibody or CTL based, could potentially be formulated such that it might result in an only transiently increased innate immune response or induce HIV-specific responses without inducing any bystander B or T cell activation (Table 2). Further studies of mucosal sites in SIV-infected natural hosts are necessary to identify any as yet uncharacterized adaptations or unknown factors that may protect from virus transmission and/or disease progression and thus shed insights into how such an adjuvant might be formulated. Finally, the recent identification of viremic nonprogressors, HIV-1-infected individuals with relatively low immune activation and persistently high $\mathrm{CD}^{+} \mathrm{T}$ cell counts, further indicates that the adaptations in the natural hosts may be relevant to HIV infection in humans ${ }^{51}$. Although the use of natural SIV hosts to inform vaccine design is still in its infancy, we believe that studies in these African nonhuman primate species will ultimately generate new approaches that may lead to the development of a successful HIV vaccine.

\section{ACKNOWLEDGMENTS}

We wish to thank A. Bernstein and N. Miller for their helpful discussions.

Published online at http://www.nature.com/naturemedicine/.

Reprints and permissions information is available online at http://npg.nature.com/ reprintsandpermissions/.

1. Fauci, A.S. et al. HIV vaccine research: the way forward. Science $\mathbf{3 2 1}, 530-532$ (2008).

2. Blankson, J.N., Persaud, D. \& Siliciano, R.F. The challenge of viral reservoirs in HIV-1 infection. Annu. Rev. Med. 53, 557-593 (2002).

3. Walker, B.D. \& Korber, B.T. Immune control of HIV: the obstacles of HLA and viral diversity. Nat. Immunol. 2, 473-475 (2001).

4. Stebbing, J., Gazzard, B. \& Douek, D.C. Where does HIV live? N. Engl. J. Med. 350, 1872-1880 (2004)

5. VandeWoude, S. \& Apetrei, C. Going wild: Lessons from T-lymphotropic naturally occurring lentiviruses. Clin. Microbiol. Rev. 19, 728-762 (2006).

6. Hahn, B.H., Shaw, G.M., De Cock, K.M. \& Sharp, P.M. AIDS as a zoonosis: scientific and public health implications. Science 287, 607-614 (2000).

7. Paiardini, M., Pandrea, I., Apetrei, C. \& Silvestri, G. Lessons learned from the natural hosts of HIV-related viruses. Annu. Rev. Med. 60, 485-495 (2009).

8. Pandrea, I., Sodora, D.L., Silvestri, G. \& Apetrei, C. Into the wild: simian immunodeficiency virus (SIV) infection in natural hosts. Trends Immunol. 29, 419-428 (2008).

9. Silvestri, G., Paiardini, M., Pandrea, I., Lederman, M.M. \& Sodora, D.L. Understanding the benign nature of SIV infection in natural hosts. J. Clin. Invest. 117, 3148-3154 (2007).

10. Pandrea, I., Silvestri, G. \& Apetrei, C. AIDS in African nonhuman primate hosts of SIVs: A new paradigm of SIV infection. Curr. HIV Res. 7, 57-72 (2009).

11. Keele, B.F. et al. Increased mortality and AIDS-like immunopathology in wild chimpanzees infected with SIVcpz. Nature advance online publication, doi:10.1038/ nature08200 (23 July 2009).

12. Sharp, P.M., Shaw, G.M. \& Hahn, B.H. Simian immunodeficiency virus infection of chimpanzees. J. Virol. 79, 3891-3902 (2005).

13. Diop, O.M. et al. High levels of viral replication during primary simian immunodeficiency virus SIVagm infection are rapidly and strongly controlled in African green monkeys. J. Virol. 74, 7538-7547 (2000).

14. Onanga, R. et al. Primary simian immunodeficiency virus SIVmnd-2 infection in mandrills (Mandrillus sphinx). J. Virol. 80, 3301-3309 (2006).

15. Kornfeld, C. et al. Antiinflammatory profiles during primary SIV infection in African green monkeys are associated with protection against AIDS. J. Clin. Invest. 115, 1082-1091 (2005).

16. Gordon, S.N. et al. Severe depletion of mucosal CD4 ${ }^{+}$T cells in AIDS-free SIV-infected sooty mangabeys. J. Immunol. 179, 3026-3034 (2007).

17. Pandrea, I.V. et al. Acute loss of intestinal $\mathrm{CD}^{+}{ }^{+} \mathrm{T}$ cells is not predictive of SIV virulence. J. Immunol. 179, 3035-3046 (2007).

18. Meythaler, M. et al. Differential CD4+ T-lymphocyte apoptosis and bystander T-cell activation in rhesus macaques and sooty mangabeys during acute simian immunodeficiency virus infection. J. Virol. 83, 572-583 (2009).
19. Lederer, S. et al. Transcriptional profiling in pathogenic and non-pathogenic SIV infections reveals significant distinctions in kinetics and tissue compartmentalization. PLoS Pathog. 5, e1000296 (2009).

20. Rey-Cuillé, M.A. et al. Simian immunodeficiency virus replicates to high levels in sooty mangabeys without inducing disease. J. Virol. 72, 3872-3886 (1998).

21. Goldstein, S. et al. Wide range of viral load in healthy African green monkeys naturally infected with simian immunodeficiency virus. J. Virol. 74, 11744-11753 (2000).

22. Broussard, S.R. et al. Simian immunodeficiency virus replicates to high levels in naturally infected African green monkeys without inducing immunologic or neurologic disease. J. Virol. 75, 2262-2275 (2001).

23. Pandrea, I. et al. High levels of SIVmnd-1 replication in chronically infected Mandrillus sphinx. Virology 317, 119-127 (2003).

24. Silvestri, G. et al. Nonpathogenic SIV infection of sooty mangabeys is characterized by limited bystander immunopathology despite chronic high-level viremia. Immunity 18, 441-452 (2003).

25. Gordon, S.N. et al. Short-lived infected cells support the bulk of virus replication in naturally SIV-infected sooty mangabeys: implications for AIDS pathogenesis. J. Virol. 82, 3725-3735 (2008).

26. Pandrea, I. et al. Simian immunodeficiency virus SIVagm dynamics in African green monkeys. J. Virol. 82, 3713-3724 (2008).

27. Dunham, R. et al. The AIDS-resistance of naturally SIV-infected sooty mangabeys is independent of cellular immunity to the virus. Blood 108, 209-217 (2006).

28. Zahn, R.C. et al. Simian Immunodeficiency Virus (SIV)-specific $\mathrm{CD} 8^{+} \mathrm{T}$ cell responses in chronically SIVagm-infected vervet African green monkeys. J. Virol. 82, 1157711588 (2008).

29. Lozano Reina, J.M. et al. Gag p27-specific B- and T-cell responses in simian immunodeficiency virus SIVagm-infected African green monkeys. J. Virol. 83, 2770-2777 (2009).

30. Wang, Z., Metcalf, B., Ribeiro, R.M., McClure, H. \& Kaur, A. $T_{H^{-1}}$-type cytotoxic CD8 ${ }^{+}$ T-lymphocyte responses to simian immunodeficiency virus (SIV) are a consistent feature of natural SIV infection in sooty mangabeys. J. Virol. 80, 2771-2783 (2006).

31. Chakrabarti, L.A. et al. Normal T-cell turnover in sooty mangabeys harboring active simian immunodeficiency virus infection. J. Virol. 74, 1209-1223 (2000).

32. Brenchley, J.M. et al. Differential $T_{H} 17$ CD4 T-cell depletion in pathogenic and nonpathogenic lentiviral infections. Blood 112, 2826-2835 (2008).

33. Brenchley, J.M. et al. Microbial translocation is a cause of systemic immune activation in chronic HIV infection. Nat. Med. 12, 1365-1371 (2006).

34. Kaur, A. et al. Dynamics of T- and B-lymphocyte turnover in a natural host of simian immunodeficiency virus. J. Virol. 82, 1084-1093 (2008).

35. Letvin, N.L. Correlates of immune protection and the development of a human immunodeficiency virus vaccine. Immunity 27, 366-369 (2007).

36. Barry, A.P. et al. Depletion of $\mathrm{CD} 8^{+}$cells in sooty mangabey monkeys naturally infected with simian immunodeficiency virus reveals limited role for immune control of virus replication in a natural host species. J. Immunol. 178, 8002-8012 (2007).

37. Milush, J.M. et al. Virally-induced $\mathrm{CD}^{+} \mathrm{T}$ cell depletion is not sufficient to induce AIDS in a natural host. J. Immunol. 179, 3047-3056 (2007).

38. Fultz, P.N., Gordon, T.P., Anderson, D.C. \& McClure, H.M. Prevalence of natura infection with simian immunodeficiency virus and simian T-cell leukemia virus type I in a breeding colony of sooty mangabey monkeys. AIDS 4, 619-625 (1990).

39. Otsyula, M.G. et al. Apparent lack of vertical transmission of simian immunodeficiency virus (SIV) in naturally infected African green monkeys, Cercopithecus aethiops. Ann. Trop. Med. Parasitol. 89, 573-576 (1995).

40. Pandrea, I. et al. Paucity of CD4+CCR5 ${ }^{+}$T-cells may prevent breastfeeding transmission of SIV in natural non-human primate hosts. J. Virol. 82, 5501-5509 (2008).

41. Pandrea, I. et al. Paucity of $\mathrm{CD} 4^{+} \mathrm{CCR} 5^{+} \mathrm{T}$ cells is a typical feature of natural SIV hosts. Blood 109, 1069-1076 (2007).

42. Estes, J.D. et al. Early resolution of acute immune activation and induction of PD-1 in SIV-infected sooty mangabeys distinguishes nonpathogenic from pathogenic infection in rhesus macaques. J. Immunol. 180, 6798-6807 (2008).

43. Mandl, J.N. et al. Divergent TLR7 and TLR9 signaling and type I interferon production distinguish pathogenic and nonpathogenic AIDS virus infections. Nat. Med. 14 1077-1087 (2008).

44. Pandrea, I. et al. Experimentally-induced immune activation in natural hosts of SIV induces significant increases in viral replication and $\mathrm{CD}^{+} \mathrm{T}$ cell depletion. $J$. Immunol. 181, 6687-6691 (2008).

45. Beaumier, C.M. et al. Downregulation of CD4 by memory CD4+ ${ }^{+}$cells in vivo renders African green monkeys resistant to progressive SIVagm infection. Nat. Med. 15 aaa-bbb (2009).

46. Schindler, M. et al. Nef-mediated suppression of T cell activation was lost in a lentiviral lineage that gave rise to HIV-1. Cell 125, 1055-1067 (2006).

47. Kirchhoff, F. Is the high virulence of HIV-1 an unfortunate coincidence of primate lentiviral evolution? Nat. Rev. Microbiol. 7, 467-476 (2009).

48. Kosub, D.A. et al. $\gamma / \delta$ T-cell functional responses differ after pathogenic human immunodeficiency virus and nonpathogenic simian immunodeficiency virus infections. J. Virol. 82, 1155-1165 (2008).

49. Klatt, N.R. et al. Availability of activated $C D 4^{+}$T cells dictates the level of viremia in naturally SIV-infected sooty mangabeys. J. Clin. Invest. 118, 2039-2049 (2008).

50. Johnson, P.R., et al. Vector-mediated gene transfer engenders long-lived neutralizing activity and protection against SIV infection in monkeys. Nat Med. 15, aaa-bbb (2009).

51. Choudhary, S.K. et al. Low immune activation despite high levels of pathogenic human immunodeficiency virus type 1 results in long-term asymptomatic disease. J. Virol. 81, 8838-8842 (2007). 